\title{
The study of the velocity field of the air flowing the swirl diffusers using PIV method.
}

\author{
Marek Jaszczur ${ }^{1 *}$, Marek Borowski ${ }^{2}$, Michat $\mathrm{Karch}^{2}$, Marian Branny ${ }^{2}$ \\ ${ }^{1}$ Faculty of Energy and Fuels, AGH University of Science and Technology, 30-059 Krakow, Poland, \\ ${ }^{2}$ Faculty of Mining and Geoengineering, AGH University of Science and Technology, 30-059 Krakow, Poland
}

\begin{abstract}
The geometry of swirl diffusers plays a primary role in the effective airflow distribution. In this work, the airflow of swirl diffusers has been studied with the use of the Particle Image Velocimetry (PIV) method in order to analyse the mean swirling air flow and the geometry influence on the results. The presented laboratory model was a simplification of the real system and was made in the geometric scale 1:10. Based on the measurements, the velocity flow field was evaluated and the range of the effective operation of the diffuser in the axis of the flow was determined. This will enable a better definition of the conditions of ventilation in buildings and will help in the design of more efficient diffusers. Also, it will help in a better understanding of the swirl diffuser airflow and the physical effects of the key geometric features.
\end{abstract}

\section{Introduction}

Air swirl diffusers are popular for ceiling level air supply systems and they have been widely used for Indoor Air Quality (IAQ) and the occupant's comfort for more than three decades. They are nowadays among the most popular diffusers commonly used in air-conditioning systems. In the case of swirl diffusers, the fluid flow is usually significantly influenced by the characteristics of different diffuser designs. At the stage of the ventilation system design, a typical focus is on the ventilation effectiveness and it takes into account a large number of parameters. However, a discrepancy between the design and the practice is often experienced, mainly because the operating conditions do not correspond to the designing conditions.

The typical project validation methods are: the point-by-point method (anemometers methods), where detectors are moved randomly around the ventilated space, particle streak velocimetry (PSV), schlieren photography, particle tracking velocimetry (PTV) and particle image velocimetry (PIV) [9].

For the design of the air distribution in the room, it is necessary to know the distribution of the velocity field and the velocity profile which is generated by the swirl diffusers. This will enable a better definition of the conditions of ventilation in buildings. The existing anemometric methods make it possible only to determine the approximate range of operation of the diffuser. The information about the velocity field is required in order to determine the range of the diffuser's effective operation in the flow axis.

In the literature, several methods of fluid flow modelling in ventilation systems with diffusers have been proposed. Computational Fluid Dynamics is nowadays one of the most useful techniques used to predict the air distribution inside a ventilated space. However, it is known that the accuracy of CFD predictions is very sensitive to the boundary conditions assumed and supplied. On the other hand, due to the large scale differences between the diffuser size and the space dimension, it is common to simplify the geometric model of the diffuser. That, in combination with inadequate turbulence modelling, may generate a result which is far from the actual one. A CFD simulation needs to specify accurately the flow, the thermal and species conditions as well as the flow conditions, in order to ensure the correct heat momentum and the mass flow rate. In general, the approaches proposed in the literature $[1,2,11,12]$ can be categorised into: direct description, simplified geometry, box, momentum and prescribed velocity methods.

The swirl method of air distribution causes an increased induction and a fast unification of the air temperature in the zones occupied by people. The presented diffusers are designed for applications in ventilation systems for low and medium pressures. This type of diffusers is especially recommended for large spaces with heights from 3 to 12 meters.

In the process of the installation design, an important issue is the proper selection of the type and details characteristic of the diffusers. The selection is based on determining the range of the air stream. The literature extensively presents the results of analyses of the flow of air through swirl diffusers with the use of CFD $[3,4,5]$. There are a few examples of experimental research related to this topic $[6,7,13]$.

In the presented paper, Particle Image Velocimetry (PIV) has been used to investigate flow fields. The presented laboratory model was a simplification of the real system and was made in the geometric scale 1:10. The air flow conditions were adjusted to a reduced scale. Measurements were made for

\footnotetext{
* Corresponding author: marek.jaszczur@agh.edu.pl
} 
various air flow speeds and for three different angles of the diffuser blades: $45^{\circ}, 60^{\circ}$ and $75^{\circ}$. Based on the measurements, the vector velocity field and the effective operation of the diffuser were determined.

\section{Experimental setup}

In Fig.1, a photograph of the test section (swirl diffuser, laser and two cameras) for swirl diffusers has been shown. The experimental measurement was performed with the use of the mono and stereo PIV technique $[8,9,10]$. The diffuser was installed at the end of a long circular channel. In order to apply different flow rates and to stabilise the flow rate at the main fan, a frequency inverter was used together with a high accuracy calibrated orifice and anemometry.

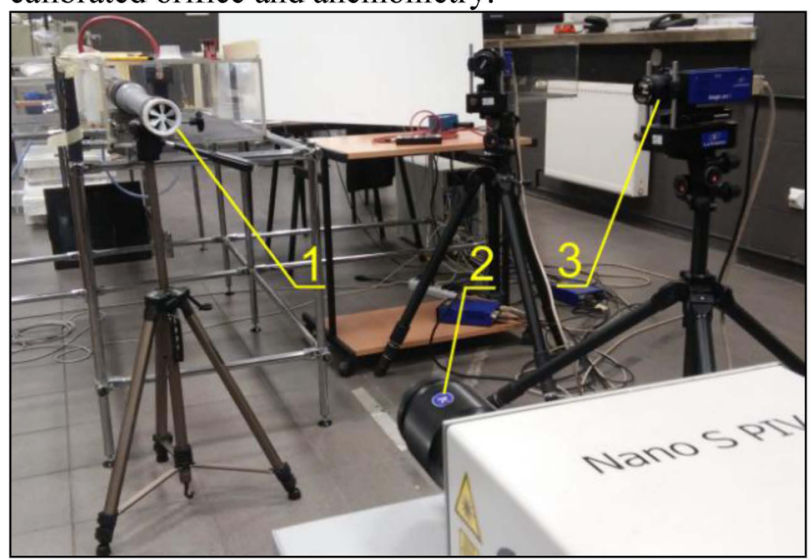

Fig. 1. Set-up of the measurement section: 1-swirl diffuser, 2laser Nd:YAG, 3-camera CCD.

As the seeding for the particle image velocimetry method, DEHS oil was used, sprayed through a high accuracy atomiser, in order to obtain particles with the diameter of about $1 \mu \mathrm{m}$. The seeding particles were introduced to the main air stream. The test section plane was illuminated by two-impulse laser $\mathrm{Nd}$ :YAG and the particle images were registered by a monochromatic CCD $(2014 \times 2048$ pixels $)$ camera.

The test procedure was preceded by the calibration with the use of a $3 \mathrm{D}$ calibrating plate. In order to obtain accurate first order and second order fluid flow statistics, it was necessary to determine the number of the required non-correlated flow images. It was found that at least 1000 fields (double picture frames) are necessary for one flow field analysis.

Based on several tests and an analysis of the optimal interrogation window for the velocity vectors, evaluation by the cross-correlation method was performed. It was found that the best interrogation window is a dynamic window in the range between $32 \times 32$ and $64 \times 64$ pixels. The Davis 7 software was used to analyse the images and to find the velocity vector components.

It was decided to experimentally determine the velocity field of the air flowing through the swirl diffusers. In this measurement of the airflow in a laboratory model of swirl diffusers, the geometrical scale of the physical model was 1:10 and the measurements were performed for steady and isothermal flow conditions.

\section{Measurement}

In this research, three different models of swirl diffusers were used, i.e. with the blade angles of $45^{\circ}, 60^{\circ}$ and $75^{\circ}$ (see Fig.2 for reference). The diameter of the diffuser's model at the diffuser's inlet was $50 \mathrm{~mm}$, which corresponds to the actual $500 \mathrm{~mm}$ diffuser. The diffusers were tested at four different volumetric flow rates of the air: $100,185,310$ and $380 \mathrm{~m}^{3} / \mathrm{h}$, and these values were measured at the fan inlet section with the use of anemometry.

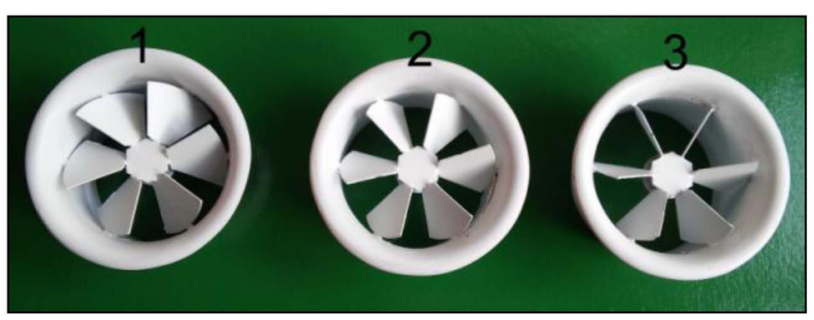

Fig. 2. Setting diffuser vanes: angle of 45 degrees (1), angle of 60 degrees (2) and angle of 75 degrees (3).

\section{Results}

The applied method enables the obtaining of images of the vector field velocity in the studied area at once. On this basis, the flow velocity can be analysed at any point of the test section. Fig. 3 shows an image of the measured velocity field and the velocity profiles created on the basis of this image. The presented results were obtained for the $45^{\circ}$ diffuser and for the flow rate of $380 \mathrm{~m}^{3} / \mathrm{h}$.

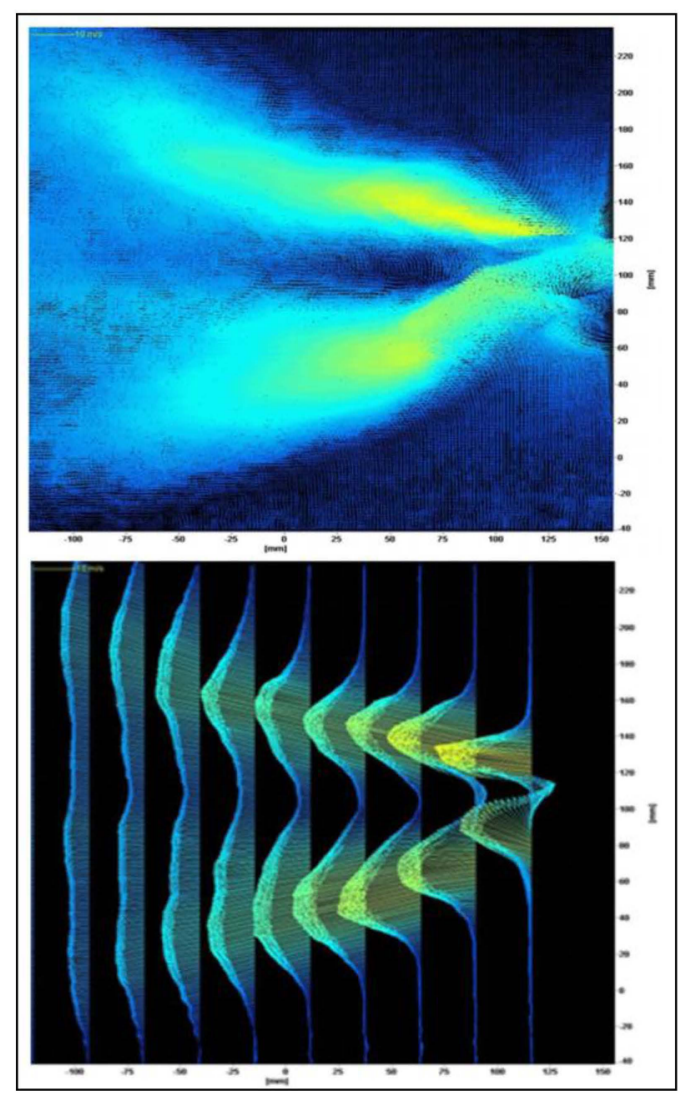

Fig. 3. The image of the measured velocity field (top) and velocity profiles (bottom). For the case: $45^{\circ}$ diffuser and flow rate $380 \mathrm{~m}^{3} / \mathrm{h}$. 
In Fig.4, the velocity vector component $V_{x}$ (axial) measured at the center-plane for the flow rate of $380 \mathrm{~m}^{3} / \mathrm{h}$ and for three different diffuser angles is presented. As can be seen, the flow dispersion of the main jet flow in the direction of the outer region is the best pronounced for the angle of 45 degrees. The direction $x$ (axial) is the leading direction in accordance with the axis of the diffuser but decreasing blades angle radial component is increasing. The range of the effective operation of the diffuser in the axial direction for high angle diffusers is very narrow and does not guarantee a proper efficient hot/cold (or fresh/used) flow mixing.

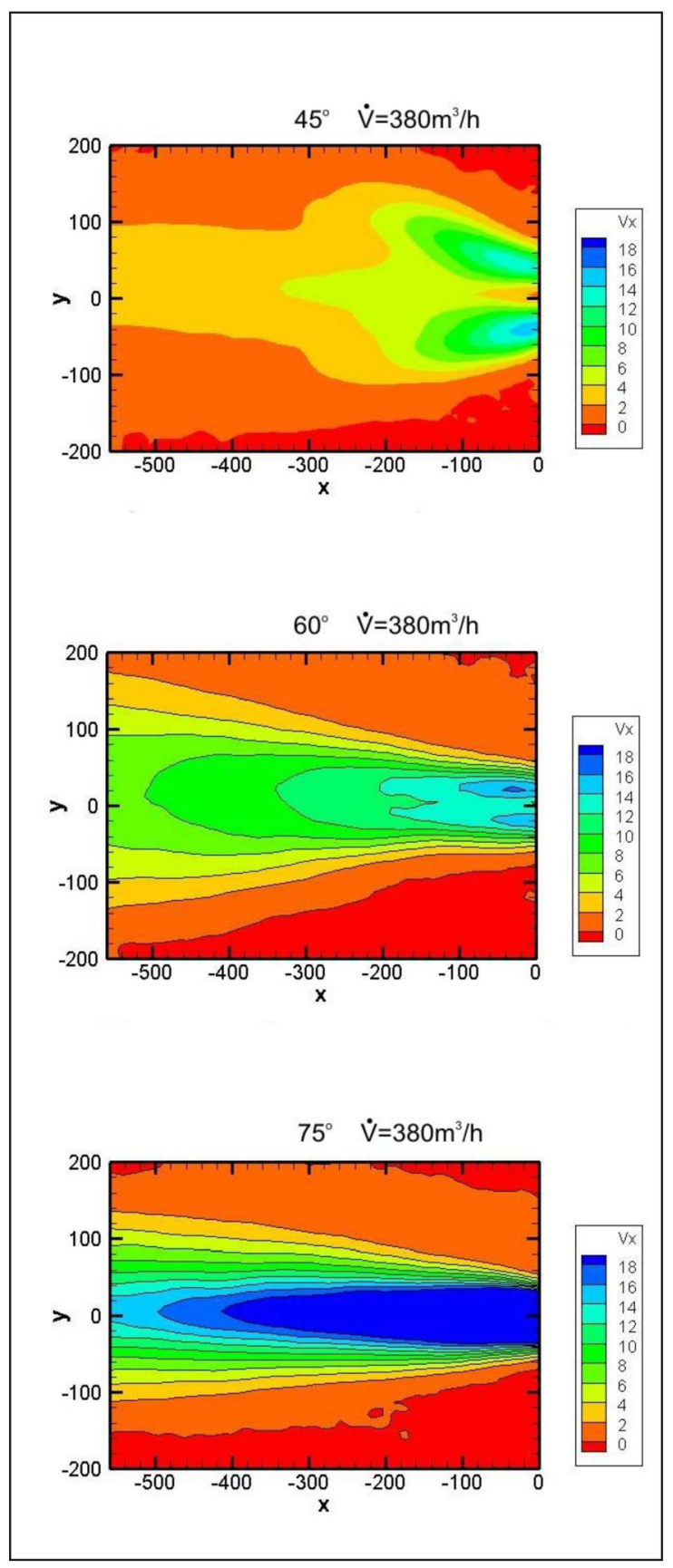

Fig. 4. The velocity vector axial component $V_{x}$ at the test section centre plane for air flow rate $385 \mathrm{~m}^{3} / \mathrm{h}$ and for different blade angles.

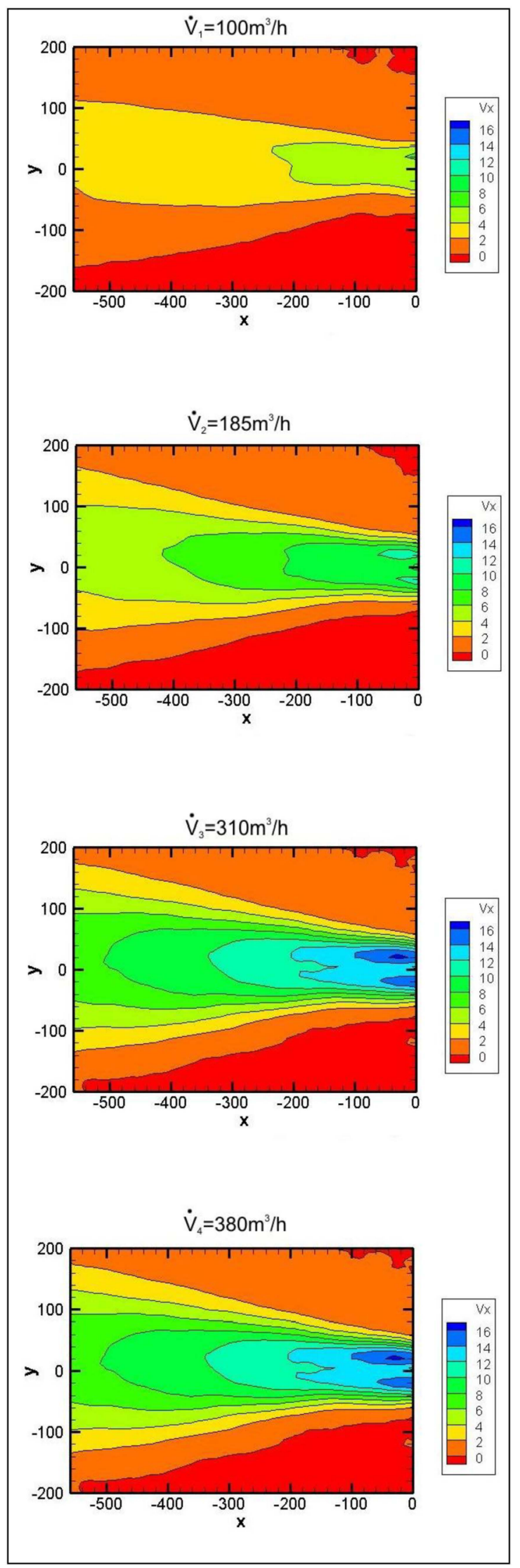

Fig. 5. The velocity vector axial component $V_{x}$ at the test section centre plane and for different air flow rates. All for the diffuser angle of 60 degrees. 
Fig.5 shows the velocity vector axial component $V_{x}$ at the test section centre plane and for four volumetric flow rates: $100,185,310$ and $380 \mathrm{~m}^{3} / \mathrm{h}$. The obtained velocity field for the diffuser angle $60^{\circ}$ is similar for different flow rates, and after normalisation it becomes almost identical.

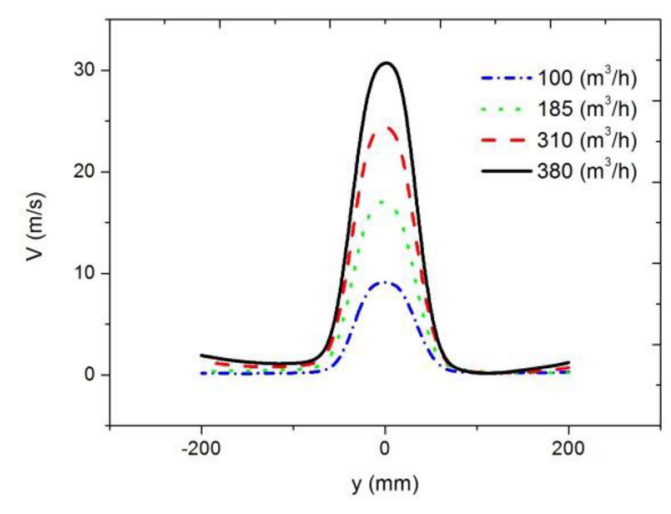

Fig. 6. Velocity magnitude profiles $2 \mathrm{~cm}$ downstream from the diffuser outlet for a $75^{\circ}$ diffuser and at different flow rates.

Fig. 6 shows the velocity for the $75^{\circ}$ diffuser and at the distance of $2 \mathrm{~cm}$ downstream from the diffuser outlet. In Fig.7, a normalised flow pattern for this diffuser is presented. It can be seen that, for a specified diffuser type, the obtained flow pattern has a similar shape.

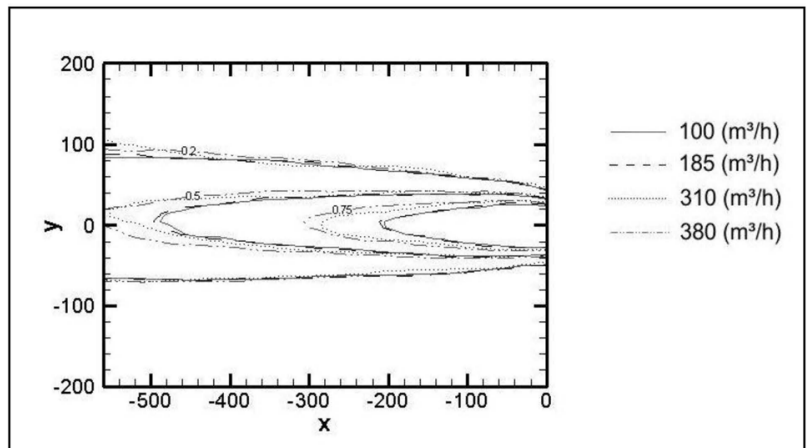

Fig. 7. The normalised velocity flow pattern for $75^{\circ}$ diffuser and for different air flow rates.

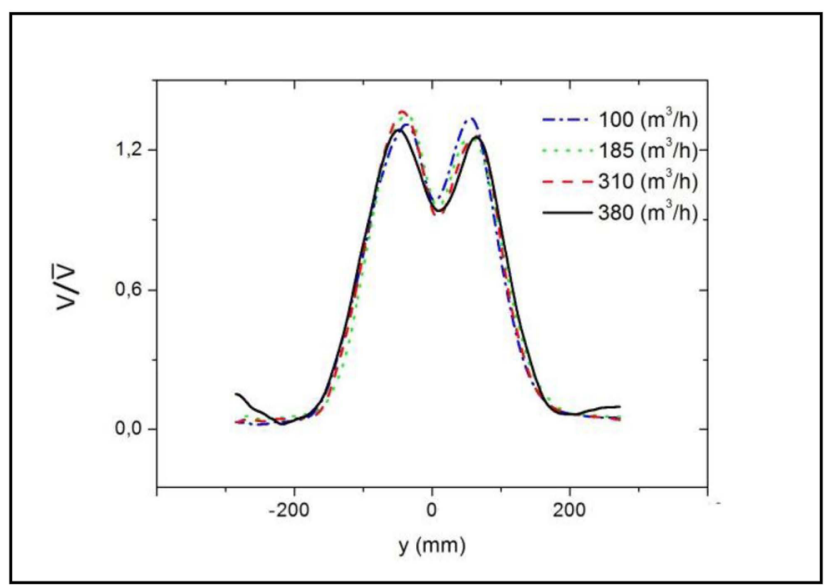

Fig. 8. The normalised velocity flow pattern for $60^{\circ}$ diffuser and for different air flow rates.

Fig.8. shows the normalised velocity flow pattern for the $60^{\circ}$ diffuser and for different air flow rates. These profiles were determined at the distance of $15 \mathrm{~cm}$ downstream from the diffuser outlet. As it is shown, the normalised profiles are quite similar.

\section{Conclusions}

The PIV method was used in order to determine the velocity field of the air flowing through a swirl diffuser. This method offers a good insight into the ventilation processes. The system is able to capture all the key features of the real flow with an accuracy higher than the one required for the needs of the ventilation practices. The knowledge of the shape of the air flow is important in order to provide adequate ventilation in people's workplaces.

An image of the velocity fields for different diffuser angles has shown that the widest air flow was obtained at the angle of 45 degrees and the narrowest air flow was observed at the angle of 75 degrees.

For a $45^{\circ}$ diffuser, two symmetrical streams were observed in a 2D plane. For other angles, this phenomenon is not observed.

As shown in Fig. 7 and 8, the normalised velocity flow pattern is similar in the range of the analysed values of the flow rates. This is an important observation because it suggests that it will be possible to indicate a universal function describing the diffuser type velocity field. At present, the research continues aiming to analyse the fluid flow turbulence components.

\section{Acknowledgements}

The research was supported by the Project No. 11.11.100.005.

\section{References}

1. W. Songling, S. Zhe, W. Zhengren, Journal of North China Electric Power University 38, 4, 81-85 (2011)

2. B.F. Yu, Z.B. Hu, M. Liu, H.L. Yang, Q.X. Kong, Y.H. Liu, Int. J. Refrig. 32, 3-20 (2009)

3. A. Sami, AL-Sanea, M.F Zedan, M.B. Al Harbi, Int. J. Therm. Sci. 59, 247-259 (2012)

4. R. Pandey, A Kumar Rao, V Yadav, IJSRD 3, 671677 (2015)

5. K.C. $\mathrm{Ng}, \mathrm{K}$. Kadirgama, E.Y.K. Ng, Energ. Buildings, 40, 774-781 (2008)

6. R. Pandey, A. Kumar Rao, V. Yadav, IJESRT, 4, 424-431 (2015)

7. M. Jaszczur, R. Nowak, J. Szmyd, M. Branny, M. Karch, W. Wodziak, J Phys Conf Ser. 318, 012029,1-8 (2011)

8. M. Raffel et al. Particle Image velocimetry: $A$ practical guide, Springer-Verlag, (2007)

9. J. Westerweel, Digital particle image velocimetry: Theory and applications Delft Uni. Press (1993)

10. LaVision Gmbh, Flow Master Manual for Davis 7.0 (2007)

11. T. Zhang, K.S. Lee, Q. Chen, Indoor Air, 19, 255267 (2009)

12. A. Ch. Hviid, S. Petersen, CLIMA 2016, proc. of the 12th REHVA World Congress (2016)

13. B. Sajadi, A. Mohebbian, M.H. Saidi, Proc.of the $2^{\text {nd }}$ Int. Conf. on Heating, Ventilation and AirConditioning, Tehran, Iran, June 1-3 (2010) 